OESOPHAGUS

\title{
Controlled prospective evaluation of the diagnostic yield of a laryngopharyngeal screening examination during upper gastrointestinal endoscopy
}

\author{
B Mullhaupt, D Jenny, S Albert, S Schmid, M Fried
}

Gut 2004;53:1232-1234. doi: 10.1136/gut.2003.030130

Background and aims: Examination of the laryngopharyngeal area is not always performed during routine upper gastrointestinal (UGI) endoscopies although older studies reported pathological findings in $0.9-3.5 \%$ of cases. The aim of our study was to prospectively evaluate the positive and negative predictive value of screening the laryngopharyngeal area during routine UGI endoscopy.

See end of article for authors' affiliations Methods: All patients undergoing elective UGI endoscopy between July 2000 and July 2001 were prospectively enrolled into the study. These patients underwent a careful structured examination of the laryngopharyngeal area, which was videotaped for later blinded review. If a pathological finding was suspected, patients were referred to the study otorhinolaryngologist for further evaluation. In all other

Correspondence to: Dr B Mullhaupt,

Department of

Gastroenterology and

Hepatology, University

Hospital Zurich,

Ramistrasse 100, $\mathrm{CH}$ -

8091 Zürich, Switzerland;

beat.muellhaupt@

DIM.USZ.ch

cases the videotapes were reviewed by the otorhinolaryngologist, who was blinded to the endoscopic findings.

Results: During the study period, 1311 endoscopies were performed in 1209 patients; 1191 were fully evaluated. In 62 endoscopies a pathology in the laryngopharyngeal area was suspected. Forty two were examined by the study otorhinolaryngologist whereas in the remaining 19 only a review of the videotape was possible. In 26 patients pathology was confirmed, corresponding to a positive predictive value of $43 \%$ and a negative predictive value of $100 \%$. In one patient $(0.08 \%)$, an early cancer of the pharyngeal region was detected whereas all other findings were benign lesions.

Revised version received 27 February 2004 Accepted for publication 3 March 2004

Conclusions: A screening examination of the laryngopharyngeal area should be part of every UGI endoscopy. A serious pathology such as an early cancer may be detected in approximately 1 in 1000 endoscopies.

\begin{abstract}
A large number of upper gastrointestinal (UGI) endoscopies are performed each year worldwide. During this procedure visualisation and examination of the laryngopharyngeal area could easily be carried out but is not routinely performed. The importance of such a screening examination has been emphasised in older studies where its effectiveness was evaluated either in asymptomatic patients undergoing laryngoscopy or screening was carried out during a bronchoscopic examination. ${ }^{12}$ Three studies, all using fibre endoscopes, have been published that evaluated the diagnostic yield of screening the laryngopharyngeal area during routine UGI endoscopy. ${ }^{3-5}$ A major drawback of these studies is however that in only one study did an otorhinolaryngologist confirm the pathologies detected by the endoscopists and no study included a control examination to review normal endoscopies. Therefore, the aim our study was to prospectively evaluate the positive and negative predictive value of a screening examination of the laryngopharyngeal area during routine UGI endoscopies using state of the art video endoscopes. All examinations were recorded on videotape for later review by a designated study otorhinolaryngologist and all patients with a suspected pathology were, in addition, referred to the study otorhinolaryngologist for a direct examination.
\end{abstract}

\section{METHODS}

All patients undergoing an elective UGI endoscopy at the University Hospital, Zurich, between July 2000 and July 2001 were included in this prospective study to evaluate the effectiveness of endoscopic screening of the hypopharyngeal and laryngeal regions during UGI endoscopy. Symptomatic patients or those with a known pathology in this area as well as all patients undergoing emergency endoscopy were excluded.

Before starting the study, all endoscopists from our unit attended a teaching session, taught by the study otorhinolaryngologist, who gave instruction on how to examine the laryngopharyngeal area, reviewing the most common pathological findings. Furthermore, a summary of possible pathologies in this area, including a description of the study methodology, was handed out to the study endoscopists.

The most commonly used endoscopes were the Olympus GIF-XQ 140 or -Q20 140 video endoscopes. Premedication varied according to the preference of the individual endoscopist but consisted mostly of topical anaesthesia in combination with midazolam $2.5-5 \mathrm{mg}$ and/or meperidine 25-50 mg intravenously.

All examinations were carried in the left lateral decubitus position. Examination of the laryngopharyngeal area was performed either at the beginning or end of the examination but was mostly done at the beginning. The instrument was advanced under direct vision. First the base of the tongue, the vallecula, and the epiglottis were inspected. Further advancement of the instrument with anterior flexion allowed visualisation of the arytenoids and aryepiglottic folds. The vocal cords were observed at rest and during phonation of the word "hi". The pyriform sinus was inspected with minimal lateral deflection. If inspection of the laryngopharyngeal area was not successful initially, it was repeated in reverse order at the end of the upper gastrointestinal endoscopy. If an adequate examination of the laryngopharyngeal area was not possible, the patient was excluded from further analysis.

Abbreviations: UGI, upper gastrointestinal 
The whole examination of the laryngopharyngeal area was recorded on videotape for later blinded review by an experienced study otorhinolaryngologist. Patients with a suspected pathology in the laryngopharyngeal area were, in addition, directly referred to the otorhinolaryngologist for indirect laryngoscopy, the standard method of examination of this area. If the study otorhinolaryngologist suspected a pathological lesion during review, he arranged further examinations. The results of the first part (July 2000December 2000) were compared with those of the second part (January 2001-July 2001) of the study in order to detect a learning curve effect during the progress of the study.

\section{Statistical analysis}

Statistical analysis of the results was made using the $\chi^{2}$ test. Results are presented as median (range). A p value of $<0.05$ was considered statistically significant.

\section{RESULTS}

During the study period, 1311 endoscopies were performed in 1209 patients; 627 were male and 582 female. Median age was 53 years (range 16-93). In 94 cases an adequate examination of the laryngopharyngeal area could not be performed, generally because of excessive gagging. Therefore, a thorough examination of the laryngopharyngeal area was possible in $93 \%$ of cases. In addition, six cases were excluded because of a known pathology in the laryngopharyngeal area, in four the videotape recording did not work, and in 16 the quality of the video tape was not sufficient for a thorough evaluation by the study otorhinolaryngologist. Therefore, 1191 endoscopies were fully evaluated (fig 1).

A total of 1129 (95\%) examinations were judged to be normal by the gastroenterologists, and the study otorhinolaryngologist reviewed the videotapes and found no additional pathologies. In $62(5 \%)$ endoscopies a pathology was suspected in the laryngopharyngeal area by the endoscopist and patients were referred for further examination to the study otorhinolaryngologist. Forty two patients were directly examined whereas the remaining 19 patients refused such an examination. In $26(42 \%)$ patients, all directly examined by the study otorhinolaryngologist, the pathology was confirmed whereas in $36(58 \%)$ patients either directly examined by the study otorhinolaryngologist $(n=16)$ or after review of the videotapes $(n=20)$ no pathologies were noted. This

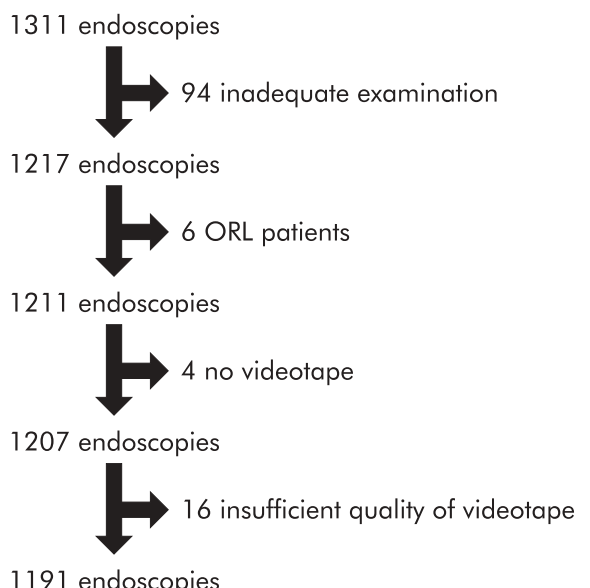

Figure 1 A total of 1311 endoscopies were performed during the study period. Reasons for exclusion were: inadequate examination in 94, known pathology in the laryngopharyngeal area in six, video recording not working in four, and insufficient quality of the videotape in 16. Therefore, 1191 endoscopies were fully evaluated. ORL patients were those with a known pathology in the laryngopharyngeal area.

\begin{tabular}{|lc|}
\hline $\begin{array}{l}\text { Table } 1 \\
\text { patients }\end{array}$ & Confirmed pathologies in the 26 \\
\hline Pathology & No of patients \\
\hline Chronic laryngitis & 7 \\
Retention cyst & 5 \\
Reinke oedema & 4 \\
Dysphonia & 3 \\
Neoplasm & 1 \\
Different minor lesions & 6 \\
Total & 26 \\
\hline
\end{tabular}

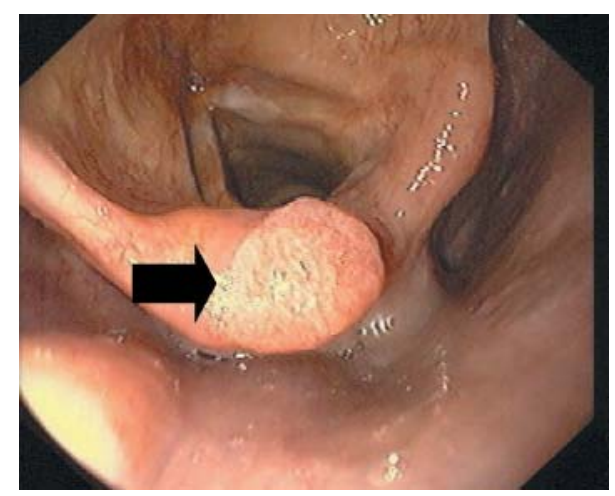

Figure 2 View of a supraglottic cancer (arrow) that was treated by $\mathrm{CO}_{2}$ laser ablation. Two years later, there is still no evidence of a recurrence.

corresponds to sensitivity, specificity, and negative and positive predictive values of $100 \%, 97 \%, 100 \%$, and $42 \%$ respectively.

The confirmed pathologies are listed in table 1. The most common findings were chronic laryngitis (seven cases), retention cysts (five cases), Reinke oedema (four cases), dysphonia (three cases), and different minor lesions (six cases). The most important finding was an early supraglottic cancer Tl NO (fig 2) which was treated by local $\mathrm{CO}_{2}$ laser ablation. Two years after treatment the patient is still tumour free.

In the first part of the study (July-December 2000), in 35 (6.6\%) of the 527 examinations a pathology was suspected by the gastroenterologist whereas in the second part of the study (January-July 01) this number decreased to 27 (4.0\%) out of 680 examinations; this difference was significant $(p=0.04)$. Also, the number of confirmed pathologies was higher in the second part of the study (second part: 13/27 (48\%); first part: $13 / 35(37 \%)$; NS).

\section{DISCUSSION}

Our study has shown that careful examination of the laryngopharyngeal area is possible during most routine endoscopies and may provide significant clinical findings. Failure to visualise the vocal cords and the associated structures was usually caused by patient retching and gagging. In the two prospective studies published so far, the success rate for visualisation of the laryngopharyngeal area ranged from $78 \%$ to $95 \% .{ }^{3}{ }^{4}$ Therefore, in the majority of upper gastrointestinal endoscopies, performed with appropriate sedation, visualisation of the laryngopharyngeal area is possible without additional patient discomfort, and little additional time is required to complete the whole examination. ${ }^{3}$

In $5 \%$ of all endoscopies a pathology was suspected by the gastroenterologist, which was finally confirmed by the 
Table 2 Compilation of the most important findings of three published studies and the current study

\begin{tabular}{lllllllll}
\hline Author & Study design & $\begin{array}{l}\text { Patient } \\
(\mathbf{n})\end{array}$ & $\begin{array}{l}\text { Mean age } \\
(\mathbf{y})\end{array}$ & $\begin{array}{l}\text { Pathologies } \\
\text { (n }(\%))\end{array}$ & $\begin{array}{l}\text { Confirmed } \\
\text { pathologies } \\
\text { (n }(\%))\end{array}$ & Cancer & False pos & False neg \\
\hline Lehman $^{3}$ & Prospective & 1120 & $\mathrm{nr}$ & $44(3.9)$ & $39(3.5)$ & 0 & $5(0.4)$ & nd \\
Kozarek $^{4}$ & Prospective & 95 & 64 & $14(14.7 \%)$ & nd & 0 & nd & nd \\
Watanabe $^{5}$ & Retrospective & 1623 & 66 & $15(0.9)$ & nd & $3(0.2)$ & nd & nd \\
Current study & Prospective & 1207 & 53 & $62(5.1)$ & $27(2.2)$ & $1(0.1)$ & $35(2.9)$ & 0 \\
\hline nr, not reported; nd, not done. & & & & & & & &
\end{tabular}

otorhinolaryngologist in $2.1 \%$ of cases. This value may slightly underestimate the real prevalence of pathologies because in 19 cases the otorhinolaryngologist could only review the videotape and did not examine the patient directly, which may reduce the sensitivity for detection of a pathology. Accordingly, the positive predictive value was higher $(62 \%)$ when only those cases where a clinical examination by the study otorhinolaryngologist was possible were considered. In our study, the number of false positive cases was significantly higher $(2.9 \%)$ compared with a previous study $(0.4 \%)$ (table 1$){ }^{3}$ However, in this study, only patients with a suspected pathology were referred for an examination and the remainder were not examined.

We detected one case of a supraglottic cancer which amounts to a cancer prevalence in the laryngopharyngeal area of approximately 1 per 1000 endoscopies. This is very similar to the prevalence of approximately 1.85 per 1000 in a previous study. ${ }^{5}$ In another study, no cancer lesions were found but two precursor lesions were detected. ${ }^{3}$ The other lesions detected in our series were benign, such as chronic laryngitis and Reinke oedema, which are nevertheless of clinical relevance.

This is the first prospective study which attempted to determine the rate of false negative examinations. In the three published studies, no such controls were included (table 2$).^{3-5}$ In contrast with the positive predictive value, the negative predictive value of our study was $100 \%$. As screening examinations should identify all patients at risk, the sensitivity and negative predictive value of a screening examination are more important statistical parameters than the positive predictive value. ${ }^{6}$ Although video review may not be as sensitive for the detection of a pathology as a direct examination, this was in our opinion the only practical way to control for false negative examinations.

The number of false positive findings was reduced by $11 \%$ in the second half of the study compared with the first half, indicating that there is a learning curve for the confident detection of laryngopharyngeal pathologies. Therefore, thorough teaching and continuous training is mandatory to achieve sufficient diagnostic accuracy in the screening of the laryngopharyngeal area during UGI endoscopy.

Gastroscopy is one to the most frequent diagnostic procedures performed. In the USA, approximately 1.5 million outpatient endoscopies are performed each year. ${ }^{7}$ Assuming a cancer detection rate of approximately 1 per 1000 endoscopies, as shown in our study, examination of the laryngopharyngeal region could lead to the detection of 1500 new potentially curable cancers. Therefore, with the little extra time needed at no extra cost and the negligible risk and discomfort, a screening examination of the laryngopharyngeal area should be an integral part of every upper oesophago-gastro-duodenoscopy.

\section{Authors' affiliations}

B Mullhaupt, S Albert, M Fried, Gastroenterology and Hepatology, University Hospital Zurich, Zurich, Switzerland

D Jenny, S Schmid, Otorhinolaryngology, Head and Neck Surgery, University Hospital Zurich, Zurich, Switzerland

\section{REFERENCES}

1 Zavala DC. Diagnostic fiberoptic bronchoscopy: Techniques and results of biopsy in 600 patients. Chest 1975;68:12-19.

2 Klein HC. Routine telescopic laryngoscopy. Am Fam Physician 1975;1 1:86-9.

3 Lehman G Compton M, Meadows J, et al. Screening examination of the larynx and pharynx during upper gastrointestinal panendoscopy. Gastrointest Endosc 1982;28:176-8.

4 Kozarek RA. Evaluation of the larynx, hypopharynx, and nasopharynx at the time of diagnostic upper gastrointestinal endoscopy. Gastrointest Endosc 1985:31:271-3.

5 Watanabe S, Matsuda K, Arima K, et al. Detection of subclinical disorders of the hypopharynx and larynx by gastrointestinal endoscopy. Endoscopy 1996;28:295-8.

6 Henkel V, Mergl R, Kohnen R, et al. Identifying depression in primary care: a comparison of different methods in a prospective cohort study. BMJ 2003;326:200-1.

7 Hall MJ, Lawrence L. Ambulatory surgery in the United States, 1995. Hyattsville, Maryland: National Center of Health Statistics, 1997:1-16.

\section{EDITOR'S QUIZ: GI SNAPSHOT}

Answer

From question on page 1219

The endoscopic picture was suggestive of ischaemic necrosis of the duodenal folds and the histological changes were consistent with ischaemic mucosal damage. Histological appearance of ischaemia in the duodenum is not pathognomic and therefore it is essential to provide the pathologist with a good history. Extensive haemoconcentration in Addisonian crisis seemed to have reduced mucosal blood circulation with subsequent necrosis. However, the endoscopic appearance after treatment underlines the pathophysiology of the mucosal damage. The patient was treated by rehydration and hydrocortisone replacement therapy and recovered well. To date, ischaemic duodenitis has been described only in patients with classical abdominal angina or severe atheromatous disease of the splanchnic arteries (Force T, MacDonald D, Eade OE, et al. Ischemic gastritis and duodenitis. Dig Dis Sci 1980;25:30710). To our knowledge, this case is the first description of ischaemic duodenitis in a patient with Addisonian crisis.

doi: $10.1136 /$ gut.2003.031286 